\title{
Trying to Show the Real Facade of Interdisciplinary Dentistry
}

\author{
Krisztina Mártha \\ University of Medicine and Pharmacy, Tîrgu Mureș, Romania
}

Beyond developing the students' professionalism, the most important mission of a dental education system is to emphasize the transdisciplinary, interdisciplinary and multidisciplinary character of dentistry. A series of studies underlined the associations between oral health and a variety of general conditions (diabetes, cardiovascular disease, pregnancy etc.), integrating oral health into chronic disease prevention efforts. ${ }^{1}$

Dental curricula has to be - and generally is - the same with the medical one, especially in the first two years of dental school. Basic anatomical, biochemical, biophysical, microbiological or physiological scientific knowledge is essential for understanding oral pathology in its complexity. ${ }^{2}$ When and if dental students or dentists will understand, that gingiva or teeth are not organs in which diseases can be separated from patient's general condition, and they should not treat only teeth or gingiva, a safe and effective practice of oral and general health can be expected.

In terms of evidence-based versus practice-oriented dentistry of the $21^{\text {st }}$ century, the $6^{\text {th }}$ edition of Dentistry Days of Tîrgu Mureș should represent a step towards professional dental treatment. Not only by the chosen topic interdisciplinary trends and innovative practices in dental medicine - , but also by the overwhelming complexity of presented lectures, we try to emphasize the links among different dental specialities and by all means, those among dentistry and medical specialities.

Krisztina Mártha • Str. Gheorghe Marinescu nr. 38, 540139 Tîrgu Mureș, Romania.

Tel: +40 265215 551. E-mail: marthakriszti@yahoo.com
In the present issue of JIM you are invited to read papers that describe different topics of interdisciplinary dental care.

In this issue, a complex review emphasizes the link between cardiovascular disease and periodontal conditions. The interaction of pro-inflammatory factors released by periodontal pathogens and vascular endothelial cells explains the role of periodonthopathogens as a cardiovascular risk factor. ${ }^{3}$ Beyond these factors, periodontopathogens are capable of hosting periodontal tissue invasion and eliciting recurrent bacteremias, and can induce preterm birth or low birthweight. ${ }^{4}$

Most part of dental treatment procedures need a welldocumented knowledge of chemical and physical properties of a large scale of dental materials, based on biochemical and biophysical curricula. A never-ending research topic seems to be the behavior of restorative materials or prosthetic restorations in oral microbiome. The stability of these conservative or prosthetic restorations depends on the patients' need of caretaking, which is a reflection of the dentist's prescribed advice.

The characteristic oral flora creates a contact surface between oral conditions and infectious diseases in general. The study of Petrovan et al. concluded that in oral and maxillofacial surgery increased Candida colonization can be expected, and as far as treatment is concerned, specific antifungal drugs should be considered. ${ }^{5}$

Willing to show the link between oral conditions and premalignant disorders, the study of Monea $e t$ al. focuses on the concomitant presence of desquamative gingivitis and periodontal health-related conditions. ${ }^{6}$ Another study finds that children diagnosed with acute lymphoblastic 
leukemia can develop different types of enamel hypoplasia, and those suffering from gastroesophageal reflux have increased caries frequency.

This congress and this focus issue should be the first step. But certainly on a road that should be followed!

\section{REFERENCES}

1. Dentists: Doctors of Oral Health. Available at: http://www.ada.org/en/ about-the-ada/dentists-doctors-of-oral-health
2. Masella RS. Renewing professionalism in dental education: overcoming the market environment. J Dent Educ. 2007;71:205-216.

3. Benedek T. The Link between Periodontal Disease, Inflammation and Atherosclerosis - an Interdisciplinary Approach. Journal of Interdisciplinary Medicine. 2017;2(S1):11-16.

4. Offenbacher S, Boggess KA, Murtha AP, et al. Progressive periodontal disease and risk of very preterm delivery. Obstet Gynecol. 2006;107:2936.

5. Petrovan C, Bereczki-Temistocle DL, Man A. Candida in Oral and Maxillo-facial Pathology: Clinical Findings and Risk Factors. Journal of Interdisciplinary Medicine. 2017;2(S1):36-42.

6. Monea M, Hănțoiu T, Stoica A, et al. The Influence of Desquamative Gingivitis on Periodontal Health. Journal of Interdisciplinary Medicine. 2017;2(S1):49-56 